\title{
A novel hydrogel-thickened microemulsion of dapsone for acne treatment: Development, characterization, physicochemical stability and ex vivo permeation studies
}

\author{
Lida Beheshti-Mall 1, Hamed Shafaroodi 2,3, Zahra Jafariazar 1, Minoo Afshar 1,* \\ 1 Department of Pharmaceutics, Pharmaceutical Sciences Branch, Islamic Azad University (IAUPS), Tehran \\ 193956466, Iran. \\ 2 Department of Pharmacology, Pharmaceutical Sciences Branch, Islamic Azad University (IAUPS), Tehran \\ 193956466, Iran. \\ 3 Department of Pharmacology, Faculty of Medicine, Tehran University of Medical Sciences, Tehran, Iran. \\ * Correspondence: afsharme@sina.tums.ac.ir (M.A.); ORCID no: 0000-0002-7209-9893
}

Received: 22 August 2017; Revised: 12 October 2017; Accepted: 15 October 2017

\begin{abstract}
Acne vulgaris is a common inflammatory skin condition, which has been reclassified as a chronic disease. The spectrum of topical acne treatments has expanded substantially in recent years and various topical medications including topical dapsone are available. Dapsone has special physicochemical properties that make its topical formulation challenging. The aim of this study was preparing a hydrogel- thickened microemulsion as a topical delivery system for dapsone. The microemulsions composed of dapsone (5\%), isopropyl myristate, tween 80 , diethylene glycol monoethyl ether, ethanol and water were prepared. The optimum microemulsion was modified with carbomer 940. Droplet size, $\mathrm{pH}$, refractive index, conductivity, rheology of the optimized formulation, and skin permeation of dapsone through rat skin were evaluated. The optimized formulation significantly increased the skin permeation of dapsone in comparison to that of control gel. Although incorporation of menthol increased the particle size, the flux of microemulgel consisting of menthol (5\%) was 2.51 times higher than that of the control. However, the skin absorption of the drug was less than $3 \%$. Six month accelerated studies proved the physicochemical stability of microemulgels. The results of this research indicate that menthol based hydrogel- thickened microemulsion system could be a promising vehicle for topical delivery of dapsone.
\end{abstract}

KEYWORDS: Dapsone; Microemulsion, Hydrogel; Menthol; Skin Permeation.

\section{INTRODUCTION}

Acne vulgaris is a common inflammatory skin condition. Although it is often considered as a selflimited disease of adolescence, its prevalence remains high into adulthood. Nearly $90 \%$ of teenagers have acne, and half of them continue to experience symptoms as adults. Therefore, there has been a recent effort to reclassify acne as a chronic disease. There are four primary pathogenic factors that interact to produce acne lesions: (i) increased and altered androgen-dependent sebum production; (ii) altered keratinization leading to comedones; (iii) Propionibacterium acnes follicular colonization; and (iv) release of inflammatory mediators into the skin. In many cases acne is often experienced as a major psychological burden $[1,2]$. Therefore, it is even more important to provide an effective as well as a safe and tolerable treatment. The spectrum of topical acne treatments has expanded substantially in recent years and various topical medications are available, ranging from azelaic acid, antibiotics, retinoids and benzoyl peroxide to several fixed combinations of these active compounds [3].

Dapsone (4,4'-diaminodiphenylsulfone), a drug of the sulfone class, has had medical applications for more than seven decades for treating various medical conditions including dermatitis herpetiformis, leprosy, and malaria. A unique property of dapsone is that it has dual therapeutic activity and demonstrates antimicrobial and anti-inflammatory properties [4]. Therefore, prior to the general acceptance of isotretinoin, oral dapsone had been reported to be effective in the treatment of nodulocystic acne. However, the potential

How to cite this article: Behesti-Mall L, Shafaroodi H, Jafariazar Z, Afshar M. A Novel Hydrogel-Thickened Microemulsion of Dapsone for Acne Treatment: Development, Characterization, Physicochemical Stability and ex vivo Permeation Studies. Marmara Pharm J. 2018; 22 (2): 267-276. 
for systemic toxicity prevented its widespread oral administration in the treatment of acne [5]. Whereas, topical dapsone $5 \%$ hydrogel, which has been marketed since late 2008, retains the anti-inflammatory benefits of dapsone while minimizing the risk of toxicity associated with systemic exposure and offers documented efficacy for the reduction of both inflammatory and non-inflammatory acne lesions [6].

Development of topical formulations for dapsone is challenging, in part because of its negligible water solubility as well as its low solubility in the oils and solvents that are established inactive ingredients in FDAapproved topical products. Although dapsone does have reasonable alcohol and glycol solubility, the addition of even small amounts of water results in the rapid and dramatic precipitation of the dissolved dapsone [7]. Moreover, because of its high lipophilicity, dapsone penetration across the stratum corneum (SC) and into viable skin layers may be a difficult task due to its affinity with SC components and tendency to be retained in this layer [8]. As microemulsions present penetration-enhancing ability and high drug loading capacity, the use of these systems for topical delivery of dapsone may overcome the aforementioned difficulties. However, the low viscosity of microemulsions limits their application in topical delivery. Several hydrocolloids, such as xanthan gum, carrageenan, and carbomer 940, have been used to increase the viscosity of microemulsions, rendering them more suitable for topical application, when compared with initial microemulsions as a vehicle for drug delivery [9]. However, some hydrogels as thickeners have been reported to change the physicochemical properties of microemulsions for topical delivery [10]. Though, nanoemulsion formulations of dapsone have been already reported, there is lack of information about the possibility of preparing hydrogel-thickened nanoemulsions and their properties especially their stability and permeability rate.

On the other hand, use of chemical skin penetration enhancers is another widely implemented approach to resolve SC barrier problem. These penetration enhancers ideally alter the physicochemical nature of SC safely and reversibly to facilitate the drug delivery through the skin [11-12]. Terpenes, naturally occurring volatile oils, appear to be clinically acceptable penetration enhancers as indicated by high penetration enhancement ability, reversible effect on the lipids of SC, minimal percutaneous irritancy at low concentrations (1-5\%) and good evidence freedom from toxicity [13]. Among different terpenes, menthol a monocyclic monoterpene fragrance free from toxic effects, has been approved to enhance the skin permeation of several hydrophilic and lipophilic drugs [11, 14].

The present study investigated the development and physicochemical evaluation of microemulsionbased gel containing insoluble dapsone (5\%) as a novel topical formulation for acne treatment. An ex-vivo permeability study using exited rat skin was conducted to assess the ability of using this system to improve skin penetration of dapsone and to study the effect of menthol as permeation enhancer. The six-month physicochemical stability of the selected formulations was also investigated.

\section{RESULTS}

\subsection{Determination of saturation solubility of dapsone in different oils and selection of microemulsion components}

To develop a microemulsion formulation for poorly water soluble dapsone, the optimum oil, surfactant and co-surfactant should be selected. Higher solubility of the drug in the oil phase is an important criterion, as it would help the microemulsion to maintain the drug in solubilized form. The solubility of dapsone in IPM and OA was calculated to be $4.75 \pm 0.30$ and $5.03 \pm 0.87 \mathrm{mg} / \mathrm{g}$, respectively. As it can be observed, there is no significant difference in solubility of dapsone in these oils, both of which have been shown to have well- known permeation-enhancing property and biocompatibility [15]. Moreover, oleic acid has been reported to be a multifunctional antimicrobial therapy agent for acne vulgaris treatment [16]. Tween 80 and tween 20 were used as surfactant because of their nonionic nature and good miscibility with IPM and OA. Since Tween 80 and tween 20 are nonionic surfactants, they are less toxic and are less affected by $\mathrm{pH}$ and change in ionic strength. Borges et al [4]. showed that a microemulsion containing IPM and isobutanol as oil phase and tween $80 /$ span 20 as surfactant was not a proper system to incorporate dapsone at the level of $5 \%$. Therefore, in this study a mixture of DEGEE, a potent dapsone solubilizer, and ethanol was used as co-surfactant. Furthermore, incorporation of ethanol to this formulation had the added advantage of preparing a selfpreserving topical dosage form.

\subsection{Construction of microemulsion phase diagrams}

The pseudoternary phase diagrams of microemulsion systems after equilibrium at room temperature were presented in (Figure 1.) The shade areas of the pseudoternary phase diagrams referred to the microemulsion regions while the outside (white) areas indicated the turbid regions. As it is shown in Figure 1 
the microemulsion zone related to OA phase diagram is grosser than that of IPM. The constructions of these phase diagrams were performed by simple visual inspection. All microemulsions were isotropic; transparent and no phase separation could be observed with all the ratios of oil/surfactant/co-surfactant/water after centrifugation at $6000 \mathrm{rpm}$ for $30 \mathrm{~min}$ at $25^{\circ} \mathrm{C}$. In addition, there were no changes in the appearance of microemulsions and areas on the diagrams after 1 month storage.

a

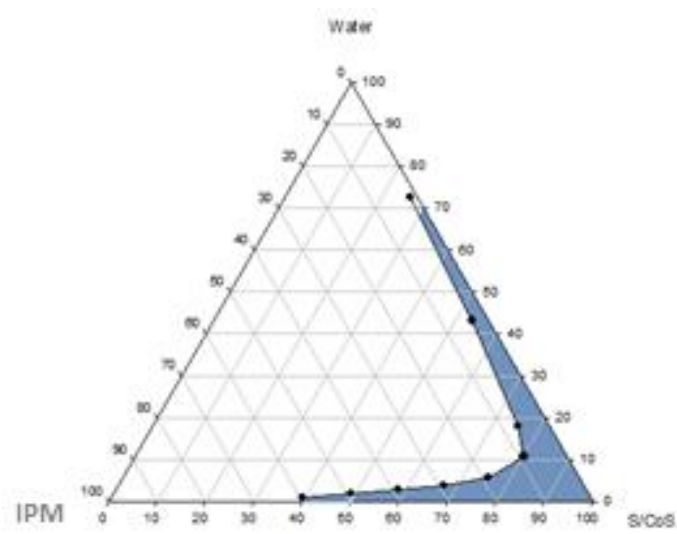

b

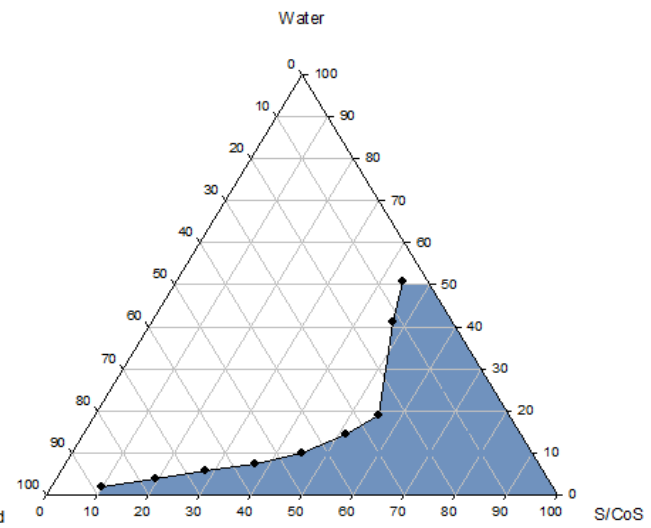

Figure 1. Pseudo-ternary phase diagrams of IPM- (a) and OA- based microemulsion systems (b).

\subsection{Characterization of the prepared microemulsions}

Depending on the physicochemical properties of the active substance, different types of microemulsion can be the optimal carrier. Considering this fact that lipophilic drugs are preferably solubilized in o/w microemulsions whereas $\mathrm{w} / \mathrm{o}$ systems seem to be the better choice for hydrophilic drugs [17], two representative microemulsion formulations (referred to M1 and M2) were selected from the microemulsion region to be incorporated with dapsone for further studies. These formulations are shown in Table 1.

Table 1. Composition of the microemulsion formulations $(\% \mathrm{w} / \mathrm{w})$.

\begin{tabular}{llllllllll}
\hline Formulation & Dapsone & IPM $^{*}$ & OA $^{* *}$ & DEGEE & Ethanol & Tween 80 & Tween 20 & Water & Menthol \\
\hline M1 & 5.15 & 4.00 & --- & 20.00 & 18.00 & 18.00 & --- & 34.85 & -- \\
M2 & 5.15 & --- & 5.00 & 20.00 & 27.00 & --- & 20.00 & 22.85 & -- \\
M3 & 5.15 & 4.00 & --- & 20.00 & 18.00 & 18.00 & --- & 32.35 & 2.50 \\
M4 & 5.15 & 4.00 & --- & 20.00 & 18.00 & 18.00 & --- & 29.85 & 5.00 \\
\hline
\end{tabular}

* Isopropyl myristate; **Oleic acid; *** Diethylene glycol monoethyl ether

\subsection{Particle size determination}

The droplet size of M1 ranged from 9.6 to $112 \mathrm{~nm}$ and the mean diameter of droplets was $48.3 \mathrm{~nm}$. Whereas, just $50 \%$ of the droplets formed in M2 system were in the range of $0.4-208 \mathrm{~nm}$. Preparing the formulation from other parts of microemulsion zone, increasing the mixing rate up to $25000 \mathrm{rpm}$ and sonicating did not resolve this problem. Therefore, M2 was dropped from further considerations and menthol at the concentration levels of 2.5 and 5\% was incorporated into M1 to form M3 and M4 formulations, respectively. The compositions of these formulations are illustrated in Table 1.

The addition of menthol to the system forming M3 and M4 (Table 2) caused an increase in the droplet size compared to M1. This could be due to the hydrophobicity of menthol and its solubility in ethanol. As it is known, co-surfactants are added into microemulsion systems to reduce the concentration of surfactant by forming a flexible film at the interfacial layer, reduce the interfacial tension and increase the fluidity of the interface. Therefore, the enlargement of the droplet size in M3 and M4 systems could be related to the localization of some menthol molecules in the interfacial surfactant film. The results of particle size analyzing for the optimized formulations are shown in Table 3. 
Table 2. Composition of modified microemulsions and control gel.

\begin{tabular}{lllllllllll}
\hline Formulation & $\begin{array}{l}\text { Dapsone } \\
(\mathrm{g})\end{array}$ & $\begin{array}{l}\text { IPM }^{*} \\
(\mathrm{~g})\end{array}$ & $\begin{array}{l}\text { DEGEE** } \\
(\mathrm{g})\end{array}$ & $\begin{array}{l}\text { Ethanol } \\
(\mathrm{g})\end{array}$ & $\begin{array}{l}\text { Tween } \\
80(\mathrm{~g})\end{array}$ & $\begin{array}{l}\text { Water } \\
(\mathrm{g})\end{array}$ & $\begin{array}{l}\text { Menthol } \\
(\mathrm{g})\end{array}$ & $\begin{array}{l}\text { Carbomer } \\
940(\mathrm{~g})\end{array}$ & $\begin{array}{l}\text { Glycerin } \\
\text { Triethanol- } \\
\text { amine }\end{array}$ \\
\hline MG1 & 5.15 & 4.00 & 20.00 & 18.00 & 18.00 & 34.35 & --- & 0.50 & --- & --- \\
MG3 & 5.15 & 4.00 & 20.00 & 18.00 & 18.00 & 31.85 & 2.50 & 0.50 & --- & --- \\
MG4 & 5.15 & 4.00 & 20.00 & 18.00 & 18.00 & 29.35 & 5.00 & 0.50 & -- & -- \\
Gel & 5.15 & --- & 20.00 & 32.60 & --- & 16.50 & --- & 0.75 & 20 & q.s \\
\hline \multicolumn{7}{r}{${ }^{*}$ Isopropyl myristate; ${ }^{* *}$ Diethylene glycol monoethyl ether }
\end{tabular}

\section{5. $\mathrm{pH}$ and conductivity measurements}

The $\mathrm{pH}$ of the prepared formulations ranged between 5.46 and 5.80, which is within the required physiologic $\mathrm{pH}$ range accepted for dermal preparations (4.0-7.0 $\mathrm{pH}$ units). The selected $\mathrm{o} / \mathrm{w}$ microemulsion formulations have conductivity value in the range of 25.40-28.25. It has been previously reported that the conductivity of $\mathrm{w} / \mathrm{o}$ microemulsions was lower than $10 \mu \mathrm{S} / \mathrm{cm}$, and the $\mathrm{o} / \mathrm{w}$ microemulsion have relatively high conductivity as compared with w/o microemulsions (about 10-100 $\mu \mathrm{S} / \mathrm{cm}$ ) [18]. Therefore, M1, M3 and M4 could be classified as $\mathrm{o} / \mathrm{w}$ microemulsion. The results of $\mathrm{pH}$ and conductivity measurements for the optimized formulations are summarized in Table 3.

Table 3. Evaluation of microemulsion M1 and M4 liquids and gel Formulations at the time of formulation.

\begin{tabular}{cccccc}
\hline Formulations & $\mathrm{pH}$ & Refractive index & $\begin{array}{c}\text { Conductivity } \\
(\mu \mathrm{S} / \mathrm{cm})\end{array}$ & $\begin{array}{c}\text { Drug content } \\
(\%)\end{array}$ & $\begin{array}{c}\text { Particle size } \\
(\mathrm{nm})\end{array}$ \\
\hline M1 & $5.80 \pm 0.10$ & $1.415 \pm 0.001$ & $25.40 \pm 0.01$ & $99.80 \pm 1.25$ & $9.60-112$ \\
M4 & $5.60 \pm 0.03$ & $1.423 \pm 0.004$ & $27.35 \pm 0.09$ & $99.54 \pm 2.82$ & $46.37 \%:$ \\
& & & & & $14.6-208$ \\
MG1 & $5.46 \pm 0.08$ & $1.413 \pm 0.002$ & $25.83 \pm 0.02$ & $99.40 \pm 0.08$ & --- \\
MG4 & $5.50 \pm 0.02$ & $1.424 \pm 0.004$ & $27.28 \pm 0.01$ & $100.00 \pm 1.75$ & --- \\
\hline
\end{tabular}

\subsection{Refractive index}

As illustrated in Table 3, the values of refractive index of optimized drug loaded formulations were found to be in the range of 1.412 to 1.424 . The higher refractive index of the microemulsion systems in comparison to that of water (1.333) could be due to the presence of DEGEE (the refractive index: 1.426-1.428), which is completely miscible with water, in the external phase of the microemulsion.

\subsection{Preparation of dapsone microemulgel}

Among different gelling agents were used, just carbomer 940 (0.5\%) formed a viscous mixture, which turned transparent within few minutes due to the formation of the microemulsion, while there was no need to neutralize the polymer to modify the viscosity. The others could not increase the viscosity of the formulations. The compositions of the modified microemulsions are shown in Table 2.

\subsection{Thermodynamic stability of dapsone microemulgel}

Visual examination showed that all the dapsone microemulgel systems were stable after being subjected to centrifugation.

\subsection{Rheological study}

Rheology is an important parameter as it affects the spreadability of the topical formulations to the skin surface. Rheological behavior of the microemulgel formulations exhibited non-Newtonian shear thinning pseudo plastic type of flow, i.e. decreases in viscosity at increasing angular velocity [19]. As the shear stress is increased (Figure 2.), the disarranged molecules of the gelling material are caused to orient their long axes in the direction of flow. Such orientation reduces the internal resistance of the material and decreases viscosity.

\subsection{Ex-vivo skin permeation studies}

Ex- vivo skin permeation studies were performed to compare the drug permeation parameters of developed microemulgels (MG1, MG3 and MG4) with control gel. The steady-state flux ( $\left.\mathrm{J}_{\mathrm{ss}}\right)$, enhancement ratio $\left(\mathrm{ER}_{\mathrm{flux}}\right)$ and cumulative amount of dapsone after $10 \mathrm{~h}\left(\mathrm{Q}_{10}\right)$ for each formulation are summarized in Table 4 and the permeation profiles through rat skin are shown in Figure 3. 


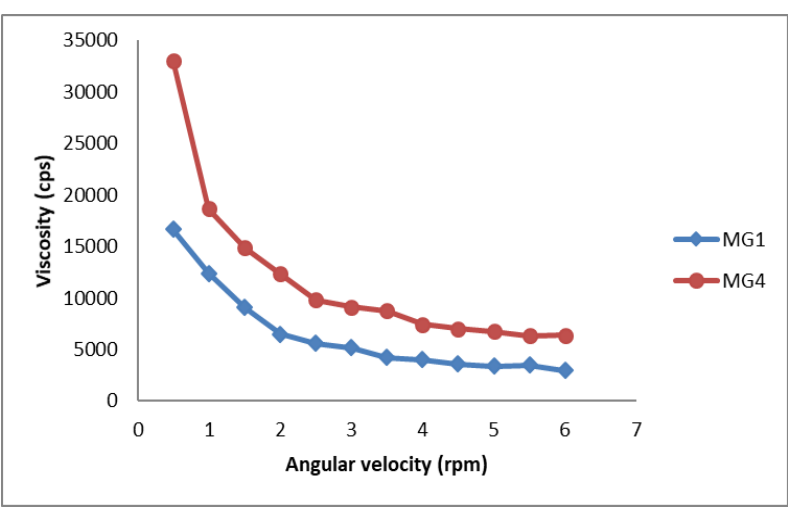

Figure 2. Rheological behavior of microemulgel formulations.

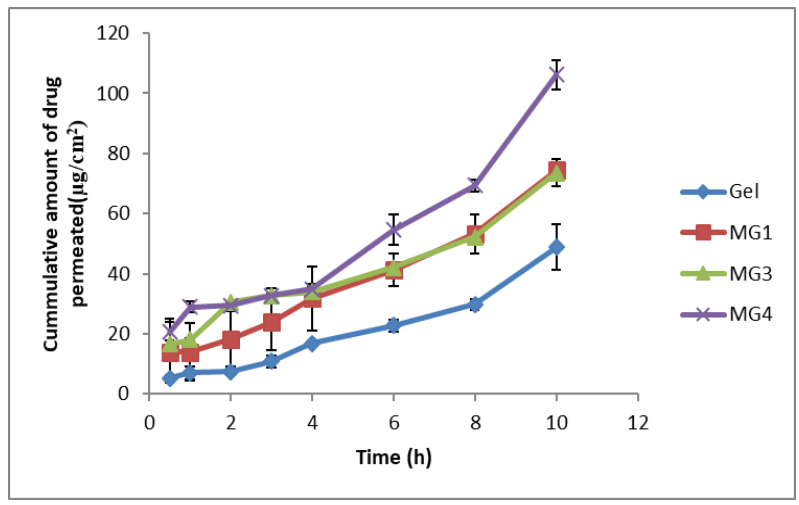

Figure 3. Mean $( \pm S D)$ amount of dapsone permeated across rat abdominal skin $(n=3)$.

The results of permeation studies showed that the rank order of ER $_{\text {flux }}$ for dapsone was as follows: MG4 $(2.51 \pm 0.55)>\operatorname{MG} 1(1.71 \pm 0.11)>$ MG3 $(1.40 \pm 0.23)$. In addition to providing highest ER flux ratio, MG4 also provided highest $Q_{10}\left(106.25 \pm 4.84 \mu \mathrm{g} / \mathrm{cm}^{2}\right)$ followed by MG1 $\left(74.38 \pm 0.70 \mu \mathrm{g} / \mathrm{cm}^{2}\right), \operatorname{MG} 3\left(73.54 \pm 4.43 \mu \mathrm{g} / \mathrm{cm}^{2}\right)$ and control gel $(48.83 \pm 7.68)$. It is worth mentioning that all formulations showed less than $3 \%$ of the drug permeation in $10 \mathrm{~h}$, which is important to be sure about non-systemic toxicity of the drug.

The calculated $\mathrm{J}_{\mathrm{ss}}$ was also highest in MG4 $\left(8.24 \pm 0.34 \mu \mathrm{g} / \mathrm{cm}^{2} \cdot \mathrm{h}\right)$, followed by MG1 $\left(5.58 \pm 0.34 \mu \mathrm{g} / \mathrm{cm}^{2}\right.$. h), MG3 (4.59 $\left.\pm 0.10 \mu \mathrm{g} / \mathrm{cm}^{2} \cdot \mathrm{h}\right)$ and lowest in control gel (3.27 \pm 0.65$)$. The $\mathrm{J}_{\text {ss }}$ and $\mathrm{Q}_{10}$ values of MG4, MG3 and MG1 were significantly higher than those of control $(p<0.05)$. Moreover, statistically significant differences ( $\mathrm{p}<0.05$ ) were found for MG1-MG4 and MG3-MG4 concerning $\mathrm{J}_{\mathrm{ss}}, \mathrm{Q}_{10}$ and $\mathrm{ER}_{\mathrm{flux}}$ values. However, no statistically significant differences were observed for MG1- MG3 for the aforementioned values.

Table 4. Permeation parameters of microemulgel systems and control gel.

\begin{tabular}{llll}
\hline Formulations & $\mathrm{J}_{\mathrm{ss}}\left(\mu \mathrm{g} / \mathrm{cm}^{2} \cdot \mathrm{h}\right)$ & $\begin{array}{l}\mathrm{Q}_{10^{*}} \\
\left(\mu \mathrm{g} / \mathrm{cm}^{2}\right)\end{array}$ & \begin{tabular}{l}
$\mathrm{ER}_{\text {flux* }}$ \\
\hline MG1
\end{tabular} \\
MG3 & $5.58 \pm 0.34$ & $74.38 \pm 0.70$ & $1.71 \pm 0.11$ \\
MG4 & $4.59 \pm 0.10$ & $73.54 \pm 4.43$ & $1.40 \pm 0.23$ \\
Gel & $8.24 \pm 0.34$ & $106.25 \pm 4.84$ & $2.51 \pm 0.55$ \\
\hline
\end{tabular}

*cumulative amount of dapsone after $10 \mathrm{~h}(\mathrm{Q} 10)$

${ }^{* *} \mathrm{ER}_{\text {flux: enhancement ratio }}$

\subsection{Stability of the selected formulations}

Stability studies were carried out to detect any changes in $\mathrm{pH}$, refractive index, droplet size, and drug content of the preparations through 6 months of storage at general and refrigerator accelerated conditions. All preparations were physically stable retaining homogeneity with no phase separation after 6 months at refrigerator accelerated condition. However, the samples were not stable at general accelerated condition (data are not shown). The stability data are summarized in Table 5.

The clarity and isotropy of all the preparations were preserved as indicated by the refractive index measurements throughout the 6-month storage period at refrigerator accelerated condition. No difference in $\mathrm{pH}$ and conductivity values was observed and decrease in drug content for gel preparations was acceptable according to ICH guidelines [20]. However, droplet size was slightly increased after 6 months. Overall, these results indicated that MG1 and MG4 formulations are sufficiently stable at refrigerator accelerated condition. 
Table 5. Evaluation of microemulsion M1 and M4 liquids and gel Formulations six months after formulation (refrigerator accelerated condition).

\begin{tabular}{cccccc}
\hline Formulations & $\mathrm{pH}$ & Refractive index & $\begin{array}{c}\text { Conductivity } \\
(\mu \mathrm{S} / \mathrm{cm})\end{array}$ & $\begin{array}{c}\text { Drug content } \\
(\%)\end{array}$ & $\begin{array}{c}\text { Particle size } \\
(\mathrm{nm})\end{array}$ \\
\hline M1 & $5.70 \pm 0.05$ & $1.416 \pm 0.004$ & $26.50 \pm 0.05$ & $90.80 \pm 2.98$ & $0.31-154$ \\
M4 & $5.55 \pm 0.10$ & $1.424 \pm 0.003$ & $28.25 \pm 0.07$ & $93.30 \pm 3.10$ & $39.44 \%:$ \\
& & & & & $3.07-208$ \\
MG1 & $5.55 \pm 0.04$ & $1.412 \pm 0.001$ & $26.15 \pm 0.01$ & $95.36 \pm 1.62$ & --- \\
MG4 & $5.45 \pm 0.10$ & $1.423 \pm 0.003$ & $27.95 \pm 0.06$ & $98.92 \pm 0.75$ & --- \\
\hline
\end{tabular}

\section{DISCUSSION}

In this study microemulgel was proposed as a carrier for topical delivery of dapsone due to its high solubilizing ability and its permeation-enhancing properties along with suitable viscosity and sustainability on the skin. A series of non-toxic commonly used components were selected for the preparation of microemulgel systems. Tween 80 is safe, very weakly irritant non-ionic surfactants with high solubilizing power [21]. DEGEE is a safe and well-tolerated solvent that has been an ingredient in cosmetic products for many years and in the last 12 years has become an FDA-approved inactive ingredient in prescription topical dermatology products. Moreover, DEGEE can act as intracutaneous depot with the ability to increase the reservoir capacity of the SC and decrease the delivery of drug to the systemic circulation, which is desirable for topical treatment of acne [7]. IPM is one of the most common ingredients in skin care products with emollient properties [22] that could be helpful to reduce skin dryness, the most common side effect of dapsone marketed topical application [23]. Moreover, it has been used previously in the anti-acne topical formulations [24]. Menthol is a safe cyclic monoterpene alcohol with a pleasant odor which possesses anti-inflammatory characteristics [25]. It was reported that menthol cause loosening of the SC lipid bilayer due to breaking of $\mathrm{H}$-bond between ceramides, which provides strength and stability to lipid bilayer and affects the barrier property of the SC. In the presence of menthol, the existing network of hydrogen bonds between ceramides may get loosened because of "competitive hydrogen bonding." This could result in penetration of water into the lipids of the SC lipid bilayer that leads to creation of new aqueous channels [13]. On the other hand, existing solubilizing agents like tween 80, ethanol and DEGEE in the o/w microemulgel formulations can increase dapsone solubility. This could be the reason of an increase in dapsone penetration despite enlargement of the particle size of the droplets in MG4 formulation. The same results have been reported previously for hydroalcoholic solutions of valsartan [26] and triptolide-loaded microemulsion [27]. Therefore, menthol as a fragrance and permeation enhancer is a suitable agent for the dapsone-loaded microemulsion.

\section{CONCLUSION}

In this study, dapsone microemulsion was successfully formulated using spontaneous microemulsification followed by being dispersed into carbomer 940-based gel matrix to form hydrogelthickened microemulsion in order to improve its solubility and skin penetration. Dermal permeation of the optimized microemulgel composed of $4 \%$ IPM, 20\% DEGEE, $18 \%$ ethanol, $18 \%$ tween $80,31.85 \%$ water and $0.5 \%$ carbomer 940 was significantly higher than that of the control gel $(\mathrm{p}<0.05)$. Moreover, incorporation of menthol (5\%) into the formulation significantly increased dermal permeation of dapsone while the skin absorption of the drug was less than $3 \%$, which is important to avoid the systemic toxicity. These formulations also showed good physicochemical stability at refrigerator accelerated condition. Therefore, the results of this research indicate that menthol based hydrogel- thickened microemulsion system could be a promising vehicle for topical delivery of dapsone.

\section{MATERIALS AND METHODS}

\subsection{Materials}

Carbomer 940 was provided by Corel Pharma Chem (Ahmedabad, India). Isopropyl myristate (IPM) was purchased from Panreac (Barcelona, Spain). Dapsone reference and raw material (97\%) were supplied by Sigma (St. Louis, MO, USA) and Merck (Darmstadt, Germany), respectively. Absolute ethanol, oleic acid (OA), formic acid, diethylene glycol monoethyl ether (DEGEE) and 1-menthol were obtained from Merck 
(Darmstadt, Germany). All other chemical and solvent were commercially available and analytical reagent grade.

\subsection{HPLC analysis}

Pharmaceutical preparations were assayed for dapsone, using an HPLC method as described in detail elsewhere [28]. This method was also used for determination of saturation solubility of dapsone in different oils. Briefly, elution was carried out on a Shimadzu HPLC system (Shimadzu, Kyoto, Japan), which was equipped with an SCL-10AVP system controller, LC-10 ADVP pump, DGU-14A degasser, and a SPD-M10AVP PDA detector. The peak areas were integrated automatically by computer using a Shimadzu Class VP software program. A $20 \mu \mathrm{L}$ volume of sample was introduced into a Rheodyne model 7725i injector.

The elution was carried out on a C18 column $(150 \mathrm{~mm} \times 4.6 \mathrm{~mm}, 5 \mu \mathrm{m}$ particle size) from Technokoroma, Spain. All analyses were performed at the column temperature of $30 \pm 1^{\circ} \mathrm{C}$ under isocratic conditions with a mobile phase of ethanol: formic acid solution $(\mathrm{pH}=3)(10: 90, \mathrm{v} / \mathrm{v})$ and a flow rate of $1.5 \mathrm{~mL} / \mathrm{min}$, using PDA detection at $210 \mathrm{~nm}$. The applied procedure was found to be linear in concentration range of $0.2-50 \mu \mathrm{g} / \mathrm{mL}\left(\mathrm{r}^{2}=0.9999\right)$. The inter-assay and intra-assay coefficients of variations were less than $2.0 \%$ and the accuracies ranged from $99.50-101.38 \%$. To be sure about the selectivity of the method, the same procedure for sample preparation was applied to the placebo samples.

\subsection{Determination of saturation solubility of dapsone in different oils}

The solubility of dapsone in IPM and OA was evaluated. For this purpose, an excess amount of drug was added to the aforementioned oils, stirred for $24 \mathrm{~h}$ at room temperature and centrifuged for $20 \mathrm{~min}$ at 10000 rpm to remove undissolved dapsone. Then, the concentration of dapsone was analyzed by HPLC.

\subsection{Construction of pseudo-ternary phase diagram}

The oil phase studied, were IPM and OA due to their availability, low cost and applicability in topical anti-acne treatments [24, 29]. Tween 80 and tween 20 were selected as surfactants (S) for IPM and OA based on the previously published microemulsion systems, respectively [30-31], while DEGEE and ethanol in the weight ratios of $(20: 18, \mathrm{w} / \mathrm{w})$ and $(20: 27, \mathrm{w} / \mathrm{w})$ for IPM and OA, respectively, were used as the co-surfactant (CoS). The weight ratio of S/CoS was 0.47 and 0.42 for for IPM and OA, respectively. The pseudoternaryphase diagrams of each oil in combination with the different S/CoS weight ratios (oil:S/Cos, 9.5:0.5-0.5:9.5) were constructed using water titration method at $25^{\circ} \mathrm{C}$. These mixtures were titrated dropwise with water under magnetic stirring. After being equilibrated, the systems were visually characterized. Transparent fluid systems were characterized as microemulsion and were constructed inside the triangular phase diagram using Sigmaplot 12 software.

\subsection{Preparation of dapsone microemulsion}

The appropriate compositions of microemulsions were chosen from the constructed phase diagrams. The drug was accurately weighted to represent $5 \%$ of the total weight of the formulation and was added to the $\operatorname{CoS}$ phase and stirred until the drug was completely dissolved (mixture A). The chosen oil was mixed with the proper surfactant (mixture B). Thereafter, mixture A was added to mixture B, stirred, and then water was incorporated into this mixture dropwise with continuous mixing.

\subsection{Preparation of dapsone microemulsion containing menthol}

Based on the results of particle size measurement, proper microemulsion was selected. Menthol was further added to the selected microemulsion at the concentration levels of $2.5 \%$ and $5 \%$ while incorporating into the CoS+drug phase.

\subsection{Preparation of dapsone gel}

Weighed quantity of carbomer 940 was added to distilled water, ethanol and glycerin and allowed to soak for $4 \mathrm{~h}$. Dapsone was solubilized in DEGEE. The latter solution was transferred to aqueous dispersion of carbomer. The mixture was stirred gradually and triethanolamine was added to neutralize the carbomer solution to form a transparent gel. The $\mathrm{pH}$ was adjusted to 6.0. 


\subsection{Characterization of dapsone microemulsion systems}

\subsubsection{Droplet Size Determination}

The size of droplets in the different dapsone microemulsion systems was determined using Nano Particle Size Analyzer (Scatter-Scope, Quidix, Seoul, South Korea) at $25 \pm 0.5^{\circ} \mathrm{C}$.

\subsection{2. $\mathrm{pH}$ and Conductivity Measurements}

The $\mathrm{pH}$ measurements of microemulgel systems were determined using a calibrated digital $\mathrm{pH}$ and conductivity meter (Mettler Toledo, Switzerland) in investigating samples. The latter experiment was performed in order to evaluate the internal structure of the systems.

\subsubsection{Refractive Index}

Refractive index of the microemulsion systems was determined at $25^{\circ} \mathrm{C}$ using RFM 80 digital refractometer (Billingham, UK).

\subsection{Preparation of dapsone microemulgel}

The optimized microemulsions were selected according to droplet size analysis. Carbomer 940, hydroxy propyl methyl cellulose, and xanthan gum were used to modify the dapsone microemulsion formulations. To prepare microemulgel systems, the gelling agent was added to water while homogenizing at $25000 \mathrm{rpm}$ to obtain homogeneous milky slurry to which the previously formulated microemulsion was added at once with constant stirring.

\subsection{Assessment of thermodynamic stability of dapsone microemulgel}

Microemulgels were centrifuged at 6,000 rpm for $30 \mathrm{~min}$ and then examined for phase separation.

\subsection{Viscosity measurement}

Viscosity was determined at $25^{\circ} \mathrm{C}$ using Brookfield digital viscometer-RVDV-III (Brookfield, Massachusetts, USA) using spindle no. 42 at different rpm(s).

\subsection{Drug content determination}

Dapsone content in liquid and jellified microemulsions was measured by dissolving known quantity of samples in ethanol by sonication. Then, the samples were diluted with HPLC-grade water and analyzed by HPLC using calibration curves of working standard solutions.

\subsection{Ex- vivo skin permeation studies}

Ex-vivo skin permeation studies were carried out on a Franz diffusion cell with an effective diffusional area of $3.46 \mathrm{~cm}^{2}$ and $30 \mathrm{ml}$ receiving chamber capacity, using rat abdominal skin. Male wistar albino rat weighing 250 $\pm 20 \mathrm{~g}$ were purchased from the Experimental Animal Center of Iran University of Medical Sciences (Tehran, Iran) for the permeation studies. The experimental protocol was approved by the Local Animal Ethical Committee of Azad University, Faculty of Pharmacy. The full thickness of rat skin was excised from the abdominal region after hair was removed with a depilatory. Subcutaneous fat and other extraneous tissues were trimmed; the skin was washed with physiological saline and then visually inspected for integrity to ensure the absence of holes or other imperfections. The prepared skin was wrapped in aluminum foil and stored in a deep freezer at $-21^{\circ} \mathrm{C}$ until further use. The excised skin samples were prepared after thawing by being hydrated in physiological saline for $60 \mathrm{~min}$ at room temperature before being clamped between the donor and the receptor chamber of Franz diffusion cells, with the SC facing the donor chamber. Then, $0.25 \mathrm{~g}$ of the dosage form was administrated on the SC. The receptor compartment was filled with $30 \%$ absolute ethanol in deionized water, and maintained at a constant temperature of $32^{\circ} \mathrm{C} \pm 0.5^{\circ} \mathrm{C}$ by a circulating-water jacket and constant magnetic stirring. Samples $(500 \mu \mathrm{l})$ were periodically drawn at suitable time intervals $(0-10$ h) from the receptor compartment and replaced with the same volume of receptor medium. All samples were analyzed spectrophotometrically at the wavelength of $292 \mathrm{~nm}$. The cumulative amount of dapsone per $\mathrm{cm}^{2}$ of rat epidermal membrane was plotted against time, and the pseudo-steady state flux $\left(\mathrm{J}_{\mathrm{ss}}\right)$ was determined from the slope of linear regression analysis. The experiments were performed in triplicate. In order to evaluate effect of microemulsion formulation and permeation enhancer, the enhancement ratio $\mathrm{ER}_{\text {flux }}$ was determined by following equation: 


$$
E R=\frac{\text { Flux from microemulgel systems }}{\text { Flux from control gel }}
$$

\subsection{Stability of the selected formulations}

Based on results of permeation studies, liquid and gel of the selected formulations were stored in wellstoppered glass containers for 6 months at $40^{\circ} \mathrm{C} \pm 2^{\circ} \mathrm{C} / 75 \%$ relative humidity (RH) $\pm 5 \% \mathrm{RH}$ (General accelerated condition) and $25^{\circ} \mathrm{C} \pm 2^{\circ} \mathrm{C} / 60 \% \mathrm{RH} \pm 5 \% \mathrm{RH}$ (refrigerator accelerated condition). The stored samples were checked for optical clarity and phase separation by visual inspection and measurement of $\mathrm{pH}$, refractive index, droplet size, and dapsone content at 0 and 6-month storage.

\subsection{Data analysis}

The characterization data were determined by three independent experiments and expressed as mean \pm S.D. One-way analysis of variance (ANOVA) test was performed to determine statistically significant differences between the data obtained from microemulsion formulations and control gel with SigmaPlot 12 software. A value of $\mathrm{p}<0.05$ was considered statistically significant. In addition, when in the analysis of variance $p$ value was significant, pair-wise comparisons were made using the Holm-Sidak post-hoc test.

Acknowledgements: This study is a part of Pharm.D. theses of Lida Beheshti-Maal. We are grateful to Poursina Pharmaceutical Co. (Tehran, Iran) for providing some of the equipment used in this study.

Author contributions: Concept - M.A, Z.J., H. SH; Design -M.A. H. SH., Z.J. ; Supervision - M.A., H. SH., Z.J.; Resource Z.J., M.A, H. SH., ; Materials - H. SH., L.BM., M.A.; Data Collection and/or Processing - M.A., L.BM., H. SH; Analysis and/or Interpretation - M.A., Z.J, L.BM., ; Literature Search - M.A., L.BM., Z.J., L.BM., ; Writing - M.A., Z.J., L.BM.; Critical Reviews - Z.J., M.A., H.SH., L.BM.

Conflict of interest statement: The authors declared no conflict of interest.

\section{REFERENCES}

[1] Dawson AL, Dellavalle RP. Acne vulgaris. BMJ. 2013; 346: f2634.

[2] Simonart T. Newer approaches to the treatment of acne vulgaris. Am J Clin Dermatol 2012; 13(6): 357-364.

[3] Tüzün Y, Wolf R, Kutlubay Z, Karakuş O, Engin B. Rosacea and rhinophyma. Clin Dermatol. 2014; 32(1): 35-46.

[4] Borges VR, Simon A, Sena AR, Cabral LM, de Sousa VP. Nanoemulsion containing dapsone for topical administration: A study of in vitro release and epidermal permeation. Int J Nanomedicine 2013; 8:535-544.

[5] Stotland M, Shalita AR, Kissling RF. Dapsone 5\% gel: A review of its efficacy and safety in the treatment of acne vulgaris. Am J Clin Dermatol. 2009; 10(4):221-227.

[6] Webster GF. Is topical dapsone safe in glucose-6-phosphate dehydrogenase-deficient and sulfonamide-allergic patients? J Drugs Dermatol. 2010; 9(5): 532-536.

[7] Osborne DW. Diethylene glycol monoethyl ether: An emerging solvent in topical dermatology products. J Cosmet Dermatol. 2011; 10(4): 324-329.

[8] Furuishi T, Kato Y, Fukami T, Suzuki T, Edno T, Nagase H, Ueda H, Tomono K. Effect of terpenes on the skin permeation of lomerizine dihydrochloride. J Pharm Pharm Sci. 2013; 16(4): 551-63.

[9] Salim N, Basri M, Rahman MB, Abdullah DK, Basri H. Modification of palm kernel oil esters nanoemulsions with hydrocolloid gum for enhanced topical delivery of ibuprofen. Int J Nanomedicine 2012; 7: 4739-4747.

[10] Mou D, Chen H, Du D, Mao C, Wan J, Xu H, Yang X. Hydrogel-thickened nanoemulsion system for topical delivery of lipophilic drugs. Int J Pharm. 2008; 353(1-2): 270-276.

[11] Shishu S, Rajan S, Kamalpreet. Development of novel microemulsion-based topical formulations of acyclovir for the treatment of cutaneous herpetic infections. AAPS PharmSciTech. 2009; 10(2): 559-565.

[12] Patel MR, Patel RB, Parikh JR, Patel BG. Novel microemulsion-based gel formulation of tazarotene for therapy of acne. Pharm Dev Technol. 2016; 21(8):921-932

[13] Tas C, Ozkan Y, Okyar A, Savaser A. In vitro and ex vivo permeation studies of etodolac from hydrophilic gels and effect of terpenes as enhancers. Drug Deliv. 2007; 14(7):453-459. 
[14] Patel NA, Patel NJ, Patel RP. Formulation and evaluation of curcumin gel for topical application. Pharm Dev Technol. 2009; 14(1): 80-89.

[15] Azeem A, Khan ZI, Aqil M, Ahmad FJ, Khar RK, Talegaonkar S. Microemulsions as a surrogate carrier for dermal drug delivery. Drug Dev Ind Pharm. 2009; 35(5): 525-547.

[16] Nakatsuji T, Kao MC, Zhang L, Zouboulis CC, Gallo RL, Huang CM. Sebum free fatty acids enhance the innate immune defense of human sebocytes by upregulating beta-defensin-2 expression. J Invest Dermatol. 2010; 130(4):985994.

[17] Junyaprasert VB, Boonme P, Songkro S, Krauel K, Rades T. Transdermal delivery of hydrophobic and hydrophilic local anesthetics from o/w and w/o Brij 97-based microemulsions. J Pharm Pharm Sci. 2007; 10(3): 288-298.

[18] Ngawhirunpat T, Worachun N, Opanasopit P, Rojanarata T, Panomsuk S. Cremophor RH40-PEG 400 microemulsions as transdermal drug delivery carrier for ketoprofen. Pharm Dev Technol. 2013; 18(4):798-803.

[19] Sohdi AA, Campbell D, Topham PD. Polymer-peptide conjugate hydrogels; towards controlled drug delivery. Chiang Mai J sci. 2012; 39(3) : 351-372.

[20] Guidance for Industry Q1A(R2) Stability Testing of New Drug Substances and Products, November 2003 ICH.

[21] El-Hadidy GN, Ibrahim HK, Mohamed MI, El-Milligi MF. Microemulsions as vehicles for topical administration of voriconazole: Formulation and in vitro evaluation. Drug Dev Ind Pharm. 2012; 38(1): 64-72.

[22] Parente ME, Gambaro A, Solana G. Study of sensory properties of emollients used in cosmetics and their correlation with physicochemical properties. J Cosmet Sci. 2005; 56(3):175-182.

[23] PDR staff. Physition Desk Reference, PDR network, 2011; pp. 598-603.

[24] Patel MR, Patel RB, Parikh JR, Patel BJ. Novel isotretinoin microemulsion-based gel for targeted topical therapy of acne: Formulation consideration, skin retention and skin irritation studies. Appl Nanosci. 2016; 6:539-553.

[25] Kamatou GP, Vermaak I, Viljoen AM, Lawrence BM. Menthol: a simple monoterpene with remarkable biological properties. Phytochemistry. 2013; 96: 15-25.

[26] Rizwan M, Aqil M, Ahad A, Sultana Y, Ali MM. Transdermal delivery of valsartan: I. Effect of various terpenes. Dev Ind Pharm. 2008; 34(6): 618-626.

[27] Chen H, Chang X, Weng T, Zhao X, Gao Z, Yang Y, Xu H, Yang X. A study of microemulsion systems for transdermal delivery of triptolide. J Control Release. 2004; 98(3):427-436.

[28] Beheshti-Maal L, Navidpour L, Afshar M. An ecofriendly and stability-indicating RP-HPLC method for determination of dapsone: Application to pharmaceutical analysis. Chiang Mai J Sci. 2016; 43(3): 420-629.

[29] Ghate VM, Lewis SA, Prabhu P, Dubey A, Patel N. Nanostructured lipid carriers for the topical delivery of tretinoin. Eur J Pharm Biopharm. 2016;108:253-261.

[30] Bhatia G, Zhou Y, Banga AK. Adapalene microemulsion for transfollicular drug delivery. J Pharm Sci. 2013; 102(8): 2622-2631.

[31] Sasivimolphan P, Lipipun V, Ritthidej G, Chitphet K, Yoshida Y, Daikoku T, Sritularak B, Likhitwitayawuid K, Pramyothin P, Hattori M, Shiraki K. Microemulsion-based oxyresveratrol for topical treatment of herpes simplex virus (HSV) infection: Physicochemical properties and efficacy in cutaneous HSV-1 infection in mice. AAPS PharmSciTech. 2012; 13(4): 1266-1275. 